\section{Audu LI \\ Mukhtar-Yola M \\ Otunete AT \\ Mairami AB \\ Nwatah VE \\ Mahmud MR \\ Ugwuanyi CU \\ Umar AU}

DOI:http://dx.doi.org/10.4314/njp.v42i3.13

Accepted: 26th March 2015

Audu LI ( $\boldsymbol{\nabla}$ )

Mukhtar-Yola M, Otunete AT

Mairami AB, Nwatah VE

Ugwuanyi CU

Department of Paediatrics,

Mahmud MR

Department of Surgery (Neurosurgery)

Umar AU

Department of radiodiagnosis,

National Hospital Abuja

Nigeria.

Email: drauduli@yahoo.com

\title{
Unexplained massive subdural haematoma in a newborn delivered by elective caesarian section: a case report
}

\begin{abstract}
Symptomatic subdural haematoma (SDH) in term infants typically occur following traumatic vaginal delivery. Emergency Caesarean Section (EmC/S) carried out after failed attempts at vaginal delivery may also be complicated by symptomatic SDH but spontaneous symptomatic SDH complicating Elective Caesarean Section $(\mathrm{ElC} / \mathrm{S})$ is a rarity. We describe a case of massive SDH in a term baby delivered by Elective
\end{abstract}

$\mathrm{C} / \mathrm{S}$ in the absence of risk factors for intracranial haemorrhage. The aim of this presentation is to highlight the significance of acute subdural haematoma in the diagnostic work up of neonates presenting with acute neurologic symptoms in the absence of traumatic delivery.

Key words: Symptomatic subdural haematoma, Caesarean section, severe birth injuries, neonatal seizure

\section{Introduction}

Subdural Hematoma is reportedly the most common type of intracranial haemorrhage encountered in the term newborn ${ }^{1}$. It is more commonly associated with vaginal delivery than operative abdominal delivery. Among babies delivered per vagina, the incidence is further increased with the use of Forceps or Vacuum extraction device $^{2}$. The speculated mechanism of SDH involves tear of the Falx and Tentorium or bridging cortical veins resulting from the increased circumferential pressure during labour and the subsequent squeezing of the head in the birth canal causing sutural overlap ${ }^{3}$. Most cases are often asymptomatic with minimal size haematoma which resolves spontaneously without any complications. Severe symptomatic SDH has been reported in babies delivered by Emergency Caesarean Section $(\mathrm{EmC} / \mathrm{S})^{4}$ but rarely in babies delivered by Elective Caesarean Section (ElC/S). The occurrence of massive symptomatic $\mathrm{SDH}$ in a term baby delivered by ElC/S prompted this report. We present a term baby delivered by elective caesarian section that was apparently normal at birth but developed acute neurologic signs (poor suck, somnolence, full and tense AF, abnormal tone and reflexes and unilateral mydriasis) on the $3^{\text {rd }}$ day of life. In the absence of a history of traumatic delivery, a clinical diagnosis of complicated intracranial infection was our initial consideration. The brain Ultrasound finding as well as the low haematocrit and blood stained CSF sub- sequently suggested intracranial bleed the location and intensity of which was confirmed with Magnetic Resonance Imaging of the brain. The cause of this haemorrhage was not known.

\section{Case Description}

A female term Neonate with a birth weight of $3.2 \mathrm{~kg}$ presented to our clinic on the $4^{\text {th }}$ day of life with a history of poor suck and somnolence which had lasted for about 24 hours. There was no history of fever, seizures, vomiting or preceding trauma. The pregnancy was supervised in a private hospital. There were no significant maternal illnesses, medications or trauma in pregnancy and antenatal ultrasound was normal. Delivery was by ElC/S conducted by a very senior Consultant Obstetrician, on account of a previous $\mathrm{C} / \mathrm{S}$ and baby cried well at birth. She received Vitamin $\mathrm{K}$ at birth, was clinically stable and sucking well until a day before presentation when a decrease in her level of physical activity was noticed. Since birth, she was cared for by her mother assisted by other relations who, despite doctor's reassurance, insisted on seeking a second opinion in a different hospital. The baby was therefore not formally referred to our hospital.

On examination, she was lethargic, pale, mildly jaundiced with a temperature of 36.8.C and SPO2 of $98 \%$. Weight on admission was $2.9 \mathrm{~kg}$ and the $\mathrm{OFC}$ was $35 \mathrm{~cm}$. 
She was not dysnoiec and had no echymotic or petechial haemorrhagic lesions. The pulse was regular at $130 / \mathrm{min}$ and there were no cardiac murmurs. Although she was conscious, the anterior fontanel was full and tense while tone and reflexes were increased in all limbs. The right pupil was about $5 \mathrm{~mm}$ dilated and non reactive while the left pupil was normal. Fundoscopy was not done. A clinical diagnosis of complicated meningitis with intracranial 'collection' was made and relevant investigations were ordered.

Lumbar puncture revealed blood stained CSF which yielded no bacterial growth while the CSF biochemistry was not reliable as a result of the blood stain. Transfontanel ultrasound showed a 'right cerebral hemispheric mass associated with marked shift of the right hemisphere to the left'. Magnetic Resonance Imaging (MRI) showed a massive SDH extending from the right frontal to the occipital area (hyper intense on T1w1, hypo intense on T2w and not suppressed on Flair), associated with shift of midline structures to the contra lateral side by about $1.22 \mathrm{~cm}$. (Fig. 1) The Haematocrit was $30 \%$, platelet count was $240 \times 10^{9}$ and white blood cell was $10.9 \times 10^{9}$. Urea and Electrolyte results were normal. Prthrombin Time and Partial Thromboplastin Time were not done.

Fig 1: Right fronto-parietal-occipital subdural haematoma with midline shift

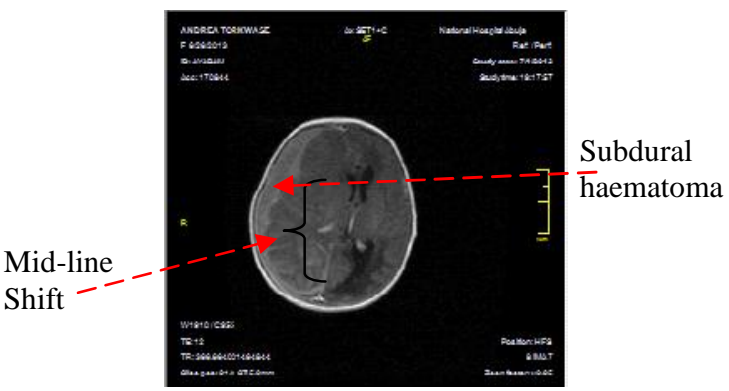

On the $3^{\text {rd }}$ day of admission, she was transfused with packed red blood cells followed by craniotomy and evacuation of $50 \mathrm{mls}$ of blood. The subdural drain was removed after 3 days. She had only a transient episode of seizure on the $2^{\text {nd }}$ post operative day, otherwise recovery was uneventful. She was treated with Phenobarbitone and antibiotics and was discharged home on the $12^{\text {th }}$ day of admission for follow-up at the Neonatal and Neurosurgical clinics. A repeat brain MRI at 3 months showed right cerebral atrophy (Fig. 2) but follow up clinical assessment has consistently demonstrated normal motor functions and age specific milestones (good neck control at 4 months, able to sit and walk without support at the age of 6months and one year respectively). The baby was last seen in the clinic at the age of 19 months and had commenced regular pre-nursery school. Hearing test (distraction test) was grossly normal and she was able to make two word sentences clearly.
Fig 2: MRI at 3 month shows right cerebral atrophy

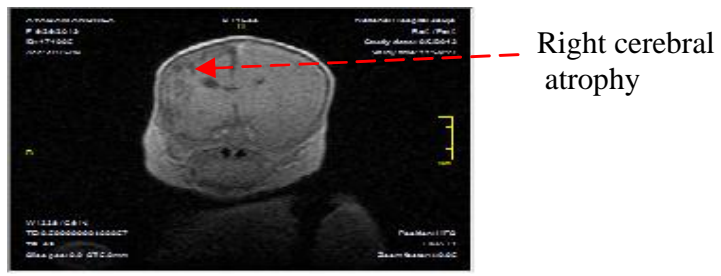

\section{Discussion}

Subdural Haematoma (SDH) is reportedly common in neonates. It is reportedly seen in $18.2 \%-46 \%$ of newborn babies in developed countries ${ }^{1,3}$ Similar prevalence studies are not readily available from Nigeria. Most cases are however asymptomatic occurring in babies following spontaneous vaginal delivery (SVD). In a prospective MRI evaluation of neonatal brains in North Carolina USA by Looney et al1 16 of $88(18.2 \%)$ asymptomatic neonates had SDH. Vaginal delivery was a significant risk factor in affected babies. The incidence of asymptomatic SDH was as high as $46 \%$ in Hawai in a prospective series reported by Rooks et al3. Spontaneous resolution usually occurs and long term neurologic complications are uncommon. On the other hand, the vast majority of symptomatic SDH in term babies are sequels of traumatic vaginal delivery with or without instrumentation $^{2,5}$.

Spontaneous SDH may occur as a result of coagulation abnormalities ${ }^{6}$ such as fetal or maternal thrombocytopenia $^{7}$ or exposure to low molecular weight heparin as reported by Bauder et $\mathrm{al}^{8}$. Although our patient was not extensively investigated for acquired or congenital haemorrhagic diathesis, the absence of bleeding from any other parts of the body or subsequent bleeding during the course of hospital stay and follow up made this an unlikely consideration. She also received a dose of intramuscular vitamin $\mathrm{K}$ at birth. Furthermore, the platelet counts of the baby and the mother were normal. Nontraumatic symptomatic SDH may also occur in critically ill neonates resulting from Hypoxic- septic insult to dural venous structures ${ }^{9}$. Our patient neither had any evidence of hypoxia at birth nor haematologic/ microbiologic evidences suggestive of sepsis.

Emergency $\mathrm{C} / \mathrm{S}$ following failed assisted labour increases the incidence of $\mathrm{C} / \mathrm{S}$ related SDH. For instance, six of the ten cases of symptomatic SDH reported by Azubuike et $\mathrm{al}^{5}$ in Nigeria, were delivered by emergency $\mathrm{C} / \mathrm{S}$ when forceps and or vacuum extraction had failed. One of the nine cases of SDH reported by Whitby et $\mathrm{al}^{10}$ from Sheffield was in a baby delivered by emergency $\mathrm{C} / \mathrm{S}$ following failed vacuum extraction. Severe maternal assault was reportedly responsible for SDH in a preterm baby delivered by emergency Caesarean section as a result of fetal distress ${ }^{11}$. It is therefore obvious that SDH when seen in babies delivered by $\mathrm{C} / \mathrm{S}$ commonly results from documented trauma before or during labour, which contrasts with the situation of the baby in our report. We however acknowledge the fact 
that the occurrence of minimal trauma during the delivery process cannot be absolutely excluded.

In a prospective series and critical review of the literature, Vinchion et al $^{12}$ stated that although SDH may occur spontaneously, non accidental head injury must always be ruled out. The absence of prenatal or parturient risk factors for SDH in our patient prompted consideration for accidental or non accidental post natal trauma which however could not be substantiated. From the foregoing, we believe our case was an unusual occurrence of severe symptomatic SDH in a term baby with no identifiable risk factors. Although the APGAR scores were not documented, the good cry at birth and the absence of symptoms in the first 3 post natal days precluded Perinatal asphyxia or significant birth related head injury.

Unlike asymptomatic SDH, acute symptomatic SDH is usually a neurological emergency that requires urgent hematoma evacuation to prevent mortality or long term neurologic morbidity ${ }^{13}$. Our patient had emergency craniotomy and haematoma evacuation done within 72 hours of admission and this we believe contributed to the favourable outcome. In the absence of a history of traumatic delivery, the differential diagnoses will include complicated meningitis, intracranial abscess and congenital cerebral malformations. Where ultrsasography or magnetic resonance imaging facilities are not available, diagnosis may be delayed and this will impact negatively on the outcome.

\section{Conclusion}

Symptomatic SDH could occur in term babies in the absence of identifiable risk factors and early cranial ultrasonography in a term baby with acute neurologic signs offers a reliable diagnostic clue. Prompt neurosurgical intervention improves long term outcome.

\section{Conflict of interest: None \\ Funding: None}

\section{References}

1. Looney CB, Smith JK, Merk LH, Wolf HM, Chescheir NC, Hamer RM, Gilmore JH. Intracranial haemorrhage in asymptomatic neonates: Prevalence on Magnetic Resonance Imaging and relationship to Obstetric and Neonatal risk factors. Radiology 2007;242:53541

2. Pollina J, Dias MS, Li V, Kachurek D, Arbesman M. Cranial birth injuries in term newborn infants. Pediatr Neurosrg 2001;35:113-119 (Medline)

3. Rooks VJ, Eaton JP, Ruess L, Petermann GW, Keck-Wherley J, Pedersen RC. Prevalence and Evolution of Intracranial Hemorrhage in Asymptomatic term Infants. Am J Neuroradiol 2008;29:1082-89

4. Azubuike JC, Izuora GI. Subdural Haematoma in Nigerian Newborns. Niger J Paediatr. 1980;7;16-19
5. Termerova J, Janota J. Severe Tentorial Haemorrhage of the term newborn with a favourable outcome. Prague Medical Report 2011;112:144-150

6. Cheung P, Obaid L, Rajani H. Spontaneous subdural hemorrhage in new born babies. Lancet. 2004;363 (9425):2001-2

7. Akman CI, Cracco J. intrauterine subdural hemorrhage. Dev Med Child Neurology 2007;42:843-846

8. Bauder F, Beinder E, Arlettaz R, Albisetti M, Boltshauser E, Gessler $P$. Intrauterine subdural hemorrhage in a preterm neonate possibly associated with maternal lowmolecular weight heparin treatment. J Perinatol 2009;9:521-523.

9. Pollamen MS. Subdural haemorrhage in infancy: keep an open eye. Forensic Sci Med Pathol. DOI 10.1007/s12024-011-9238-5
10. Whitby EH, Griffiths PD, Rutter S et al. Frequency and Natural history of subdural hemorrhages in babies and relation to obstetric factors. Lancet 2004; 846-851

11. Stephenes RP, Richardson AC, Levin JS. Bilateral Subdural Hematoma in a newborn infant. Pediatr 1997; 99:619-21.

12. Vinchon M, Delesret I, deFoortDhellememms S, Desurmont M, Noule N. Subdural hematoma in infants: can it occur spontaneously? Data from a prospective series and critical review of the literature. Child Nervous System 2010;26:1195-1205

13. Gerard C, Busl KM. Treatment of acute subdural hematoma. Current Treatment Options in Neurology. 2014 Jan;16:275 